\title{
A Note on Parallel Asynchronous Channels with Arbitrary Skews
}

\author{
Mladen Kovačević, Member, IEEE
}

\begin{abstract}
A zero-error coding scheme of asymptotic rate $\log _{2}(1+\sqrt{5})-1$ was recently described in [1] for a communication channel composed of parallel asynchronous lines satisfying the so-called no switch assumption. We prove that this is in fact the highest rate attainable, i.e., the zero-error capacity of this channel.
\end{abstract}

Index Terms-Random delay, delay injection attack, skew, shift, parallel communications, timing channel, zero-error.

\section{INTRODUCTION}

Let $C^{(A A S)}$ denote the zero-error capacity of the Asynchronous channel with an Arbitrary number of Skews (AAS); see [1, Def. 1 and Def. 2]. Let $C_{1, w}$ denote the zero-error capacity of the $(1, w)$-channel; see [1, Sec. IV]. It was recently shown in [1, Cor. 11 and Cor. 15] that

$$
\log _{2} \phi \leq C^{(A A S)} \leq \inf _{w \geq 2} C_{1, w},
$$

where $\phi=\frac{1}{2}(1+\sqrt{5})$ is the golden ratio. We prove below that $\inf _{w \geq 2} C_{1, w}=\log _{2} \phi$, thus determining the value $C^{(A A S)}$ exactly.

The $(1, w)$-channel, $w \geq 2$, is defined as follows. The transmitter sends a signal of the form

$$
S(t)=\sum_{k} a_{k} \cdot p(t-k T),
$$

and the receiver obtains

$$
R(t)=\sum_{k} a_{k} \cdot p\left(t-k T-\tau-\tau_{k}\right) .
$$

Here $a_{k} \in\{0,1\}$ are binary symbols, $T$ is the signaling interval, $p(t)$ is a pulse of duration $\leq T, \tau$ is the propagation delay, and $\tau_{k}= \pm \frac{T}{2}$ is a random deviation of the delay of each pulse 11 The channel is synchronous and the quantities $T, p(t), \tau$ are known to the receiver. Moreover, it is assumed that $\tau_{k}=+\frac{T}{2}$ when $k \equiv 1 \bmod w$ and $\tau_{k}=-\frac{T}{2}$ when $k \equiv 0 \bmod w$. In words, time is divided into groups consisting of $w$ consecutive slots (i.e., signaling intervals), and the pulses from one group cannot mix with pulses from a different group. For example, in the group of time slots $1, \ldots, w$, the pulse in slot 1 (if any) is "shifted" to the right by $\frac{T}{2}$, the pulse in slot $w$ (if any) is shifted to the left by $\frac{T}{2}$, while the pulses in slots

Date: August 28, 2017.

This work was supported by the Singapore Ministry of Education (MoE) Tier 2 grant "Network Communication with Synchronization Errors: Fundamental Limits and Codes" (Grant number R-263-000-B61-112).

The author is with the Department of Electrical \& Computer Engineering, National University of Singapore, Singapore 117583 (email: mladen.kovacevic@nus.edu.sg).

${ }^{1}$ It will be evident from the proof of Theorem 1 that the zero-error capacity of the $(1, w)$-channel is unchanged if we assume that $\tau_{k} \in\left\{-\frac{T}{2}, 0,+\frac{T}{2}\right\}$.
$2, \ldots, w-1$ are shifted either to the left or to the right by $\frac{T}{2}$, the choice being random for each pulse; the same goes for the group $w+1, \ldots, 2 w$, and so on.

For the purpose of discussing the zero-error capacity of the channel just defined, one can regard it [1] as a memoryless channel with input alphabet $\{0,1\}^{w}$. The confusability graph [4] corresponding to this channel is the graph $\Gamma_{w}$ with the vertex set $\{0,1\}^{w}$ and with an edge between any two strings $a, b \in\{0,1\}^{w}$ that are confusable in the channel, meaning that they can produce the same waveform (3) at the channel output. Evidently, $a, b$ can only be confusable if they have the same Hamming weight. Therefore, $\Gamma_{w}$ has $w+1$ connected components, one for each weight $h \in\{0,1, \ldots, w\}$.

An adjacency reducing mapping for a memoryless channel with input alphabet $\mathcal{A}$ is a mapping $f: \mathcal{A} \rightarrow \mathcal{A}$ with the property that if $a, b$ are not confusable in the channel (i.e., not adjacent in the corresponding graph $\Gamma$ ), then $f(a), f(b)$ are not confusable either. The well-known result of Shannon [4. Thm 3] states that: If there exists an adjacency reducing mapping such that its range, denoted $f(\mathcal{A})$, is an independent set in $\Gamma$, then the zero-error capacity of the channel in question equals $\log _{2}|f(\mathcal{A})|$. Clearly, in search for such mappings, one can consider each connected component of $\Gamma$ separately.

\section{THE RESULTS}

Theorem 1: $C_{1, w}=\frac{1}{w} \log _{2} F_{w}$, where $\left(F_{w}\right)$ is the Fibonacci sequencel defined by $F_{0}=1, F_{1}=2$, and $F_{w}=F_{w-1}+$ $F_{w-2}$ for $w \geq 2$.

Proof: As mentioned above, $\Gamma_{w}$ has $w+1$ connected components, one for each weight $h$, so we can consider each of these components individually. Denote by $\{0,1\}_{h}^{w}$ the set of all binary strings of length $w$ and Hamming weight $h$. Each such string $a=\left(a_{1}, \ldots, a_{w}\right)$ can be uniquely described by an $h$-tuple $x^{a}=\left(x_{1}^{a}, \ldots, x_{h}^{a}\right) \in \mathbb{Z}^{h}$ satisfying $1 \leq x_{1}^{a}<\cdots<$ $x_{h}^{a} \leq w$, where $x_{i}^{a}$ represents the position of the $i$ 'th 1 in the string $a$. For example, for $a=(1,0,0,1,0) \in\{0,1\}_{2}^{5}$, we have $x^{a}=(1,4)$. For convenience, let us subtract from each such integer $h$-tuple the vector $(1,2, \ldots, h)$ so that the resulting $h$-tuples satisfy $0 \leq x_{1}^{a} \leq \cdots \leq x_{h}^{a} \leq w-h$. Denote $\Delta_{w-h}^{h}:=\left\{x \in \mathbb{Z}^{h}: 0 \leq x_{1} \leq \cdots \leq x_{h} \leq w-h\right\}$. It is clear that $\Delta_{w-h}^{h}$ is just a different representation of $\{0,1\}_{h}^{w}$ so we shall identify the two spaces with no risk of confusion. Consequently, $\left|\Delta_{w-h}^{h}\right|=\left(\begin{array}{c}w \\ h\end{array}\right)$.

Consider $x, y \in \Delta_{w-h}^{h}$. The key observation about the confusability graph $\Gamma_{w}$ is the following: $x, y$ are confusable (i.e.,

\footnotetext{
${ }^{2}$ The initial conditions of the Fibonacci sequence are usually taken to be $F_{0}=0, F_{1}=1$, so the sequence from Theorem 1 is in fact the shifted Fibonacci sequence. We ignore this fact here for notational simplicity.
} 
$(x, y)$ is an edge of $\left.\Gamma_{w}\right)$ if and only if $\left|x_{i}-y_{i}\right| \leq 1$ for every $i \in\{1, \ldots, h\}$, i.e., if and only if $\|x-y\|_{\infty} \leq 1$. This is because each pulse of the transmitted waveform is shifted by either $+\frac{T}{2}$ or $-\frac{T}{2}$ by definition, so 1 .) if the $i$ 'th pulse of one input waveform is two or more slots away from the $i$ 'th pulse of another input waveform, then these two waveforms cannot produce the same output, and 2.) if the $i$ 'th pulse of one input waveform is at most one slot away from the $i$ 'th pulse of another input waveform, for every $i \in\{1, \ldots, h\}$, then these two waveforms can always produce the same output.

For a non-negative integer $x_{i}$ define

$$
f\left(x_{i}\right):=2\left\lfloor\frac{x_{i}}{2}\right\rfloor= \begin{cases}x_{i}, & \text { if } x_{i} \text { is even } \\ x_{i}-1, & \text { if } x_{i} \text { is odd. }\end{cases}
$$

Abusing the notation slightly define also the mapping $f: \Delta_{w-h}^{h} \rightarrow \Delta_{w-h}^{h}$ by $f(x):=\left(f\left(x_{1}\right), \ldots, f\left(x_{h}\right)\right)$. We claim that $f$ satisfies the conditions of [4, Thm 3]. Indeed, it follows from (4) that if $\left|x_{i}-y_{i}\right| \geq 2$, then $\left|f\left(x_{i}\right)-f\left(y_{i}\right)\right| \geq 2$ as well. In other words, if $x, y$ are not confusable, then $f(x), f(y)$ are not confusable either, which means that $f$ is an adjacency reducing mapping. Furthermore, the range of $f$ is an independent set in $\Gamma_{w}$, i.e., every two elements of $f\left(\Delta_{w-h}^{h}\right)$ are non-confusable. This is because $f\left(\Delta_{w-h}^{h}\right)$ contains only $h$-tuples with even entries, and so for any $x, y \in f\left(\Delta_{w-h}^{h}\right), x \neq y$, there must exist a coordinate $i$ where $\left|x_{i}-y_{i}\right| \geq 2$. This proves that $f$ satisfies the conditions of [4, Thm 3] and, therefore, the zero-error capacity of the $(1, w)$-channel equals $\frac{1}{w} \log _{2} \sum_{h=0}^{w}\left|f\left(\Delta_{w-h}^{h}\right)\right|$.

To complete the proof we need to show that $\sum_{h=0}^{w}\left|f\left(\Delta_{w-h}^{h}\right)\right|=F_{w}$. To that end we first note that $f\left(\Delta_{w-h}^{h}\right)$ can be written in the form $2 \cdot \Delta_{d}^{h}$, where $d=\left\lfloor\frac{w-h}{2}\right\rfloor$. Since $\Delta_{d}^{h}$ represents the set of binary strings of length $d+h$ and weight $h$, we get $\left|f\left(\Delta_{w-h}^{h}\right)\right|=\left|\Delta_{d}^{h}\right|=\left(h+\left\lfloor\frac{w-h}{\left.h^{2}\right\rfloor}\right)\right.$. One can now check directly that the sequence $S_{w}:=\sum_{h=0}^{w}\left(h+\left\lfloor\frac{w-h}{h}\right\rfloor\right)$ satisfies the recurrence $S_{w}=S_{w-1}+S_{w-2}$. As for the initial conditions, there is only one binary string of length $w=0$ (empty string), and there are two binary strings of length $w=$ 1 and they are non-confusable. Therefore, $S_{w}=F_{w}$.

The mapping $f$ from the above proof, and its generalizations, were used in [2], [3] to construct optimal zero-error codes for shift and timing channels.

Corollary 2: $\inf _{w>2} C_{1, w}=\lim _{w \rightarrow \infty} C_{1, w}=\log _{2} \phi$, where $\phi=\frac{1}{2}(1+\sqrt{5})$ is the golden ratio.

Proof: Theorem 1 implies $\lim _{w \rightarrow \infty} C_{1, w}=\log _{2} \phi$, so it is enough to show that $C_{1, w} \geq \log _{2} \phi$, or equivalently that $F_{w} \geq \phi^{w}$, for every $w \geq 2$. Solving the recurrence for $F_{w}$ we find that $F_{w}=\alpha \phi^{w}+(1-\alpha)\left(-\phi^{-1}\right)^{w}$, where $\alpha=\frac{4+2 \sqrt{5}}{5+\sqrt{5}} \approx$ 1.17. The statement $F_{w} \geq \phi^{w}$ is therefore equivalent to $(\alpha-1)\left(\phi^{w}-\left(-\phi^{-1}\right)^{w}\right) \geq 0$, which is easily verified to be true since $\phi^{-1}<1$.

\section{ACKNOWLEDGMENT}

The author would like to thank Vincent Y. F. Tan for reading a preliminary version of this work and providing several helpful comments.

\section{REFERENCES}

[1] S. Engelberg and O. Keren, "Reliable Communications Across Parallel Asynchronous Channels With Arbitrary Skews," IEEE Trans. Inf. Theory, vol. 63, no. 2, pp. 1120-1129, Feb. 2017.

[2] M. Kovačević and P. Popovski, "Zero-Error Capacity of a Class of Timing Channels," IEEE Trans. Inf. Theory, vol. 60, no. 11, pp. 6796-6800, Nov. 2014.

[3] M. Kovačević, M. Stojaković, and V. Y. F. Tan, "Zero-Error Capacity of $P$-ary Shift Channels and FIFO Queues," IEEE Trans. Inf. Theory, to appear. Available online: https://doi.org/10.1109/TIT.2017.2756667.

[4] C. E. Shannon, "The Zero Error Capacity of a Noisy Channel," IRE Trans. Inf. Theory, vol. 2, no. 3, pp. 8-19, Sep. 1956. 